\title{
Intra-Operative Indocyanine Green Angiography of the Parathyroid Gland
}

\author{
Jordi Vidal Fortuny ${ }^{1}$ - Wolfram Karenovics ${ }^{1} \cdot$ Frederic Triponez $^{1}$ \\ Samira M. Sadowski ${ }^{1}$
}

Published online: 4 March 2016

(C) Société Internationale de Chirurgie 2016

\begin{abstract}
Major complications of thyroid and parathyroid surgery are recurrent laryngeal nerve injuries and definitive hypoparathyroidism. The use of intra-operative Indocyanine Green Angiography for confirmation of vascular status of the parathyroid gland is reported here.
\end{abstract}

Subtotal parathyroidectomy is performed in patients with hyperparathyroidism (HPT) of renal origin (patients with renal insufficiency or after renal transplantation) and in patients with primary HPT due to hyperplasia of the four glands. The surgery involves bilateral neck exploration and identification of the four parathyroid glands. Resection of 3.5 parathyroid glands is usually performed and size of the parathyroid remnant varies according to primary disease and intra-operative findings. The main complications of subtotal parathyroidectomy are recurrent laryngeal nerve palsy, persistent HPT and definitive or transient hypoparathyroidism. Definitive hypoparathyroidism occurs if all 4 parathyroid glands are removed or if the parathyroid remnant is not well vascularized, situation that should be avoided. Until now, post-operative

A clinical series of cases using this technique was presented during the 2015 IAES meeting at the 46th World Congress of Surgery:

"Subtotal Parathyroidectomy with Indocyanine Green Angiography demonstrates good function of the parathyroid remnant".

Electronic supplementary material The online version of this article (doi:10.1007/s00268-016-3493-2) contains supplementary material, which is available to authorized users.

Samira M. Sadowski

samira.sadowski@hcuge.ch

1 Thoracic and Endocrine Surgery, University Hospitals of Geneva, 1211 Geneva, Switzerland parathyroid hormone (PTH) or intra-operative $\mathrm{PTH}$ dosage was used to verify the function of the parathyroid remnant $[1,2]$. However, the time needed to obtain PTH results after parathyroidectomy does not permit to adapt the extent of parathyroid resection intra-operatively.

In thyroid surgery, one of the main complications is post-operative hypocalcaemia, which can significantly impact quality of life. The main cause of hypocalcaemia after total thyroidectomy is hypoparathyroidism due to intra-operative damage to the parathyroid glands by trauma, inadvertent parathyroid gland removal or devascularization. The extent of damage to the parathyroid glands is difficult to predict during surgery.

A new innovative technique using intra-operative angiography with a fluorescent dye to verify perfusion of each parathyroid gland is reported here. For the first time, we illustrate a technique that allows surgeons during surgery to make sure that the parathyroid remnant is well perfused before proceeding with complete resection of the other parathyroid glands and/or to make sure that the parathyroid glands are well vascularized after thyroid resection, in order to avoid hypoparathyroidism after thyroid surgery.

Indocyanine Green (ICG) angiography was first used in the detection of macular degeneration [3]. Then the angiography was coupled with an infrared camera to search for sentinel lymph nodes, to dictate the extent of oncologic 
resections and to study hepatic function [4]. Recent studies have shown its use in bowel surgery to evaluate the vascular blood flow of anastomosis.

ICG is a 775-Dalton-sized molecule with a maximum absorption spectrum of $805 \mathrm{~nm}$ and with re-emission at $835 \mathrm{~nm}$. It is completely fixed to plasmatic proteins once in the blood stream and circulates only in the intravascular compartment. Its half-life is $3-5 \mathrm{~min}$ and elimination occurs within $15-20 \mathrm{~min}$ by the liver. ICG is mixed with $10 \mathrm{~mL}$ of sterile water, and $3.5 \mathrm{~mL}(0.3 \mathrm{mg} / \mathrm{kg})$ of it is injected intravenously. The injection can be repeated until a maximum toxic dose of $5 \mathrm{mg} / \mathrm{kg}$ per day is reached. Reactions to the molecule are rare and fatal allergies have been reported in 1/333,000 of cases. The catheter is purged after each injection for rapid image gain. After 1-2 min approximately, images are acquired using a laparoscopic PinPoint camera (Novadaq, Ontario, Canada). Using the fluorescence emitted by ICG, angiographic images are viewed on the screen in a black and white scale or as conventional images with superposed green fluorescence.
At the time of angiography with ICG, the parathyroid glands appear in shades of white and black colours, depending on the quantity of ICG flowing through parathyroid tissue, thus reflecting the degree of vascular blood flow. An imaging score for ICG was established: ICG 0: the parathyroid is black after injection of ICG, meaning that the gland is not vascularized; ICG 2: the parathyroid is white, well vascularized; ICG 1: the parathyroid is grey or heterogeneous (Fig. 1, video 1-3). During thyroid surgery, in case of discordance between visual evaluation of the gland and angiography results, a parenchymal incision is performed. If no active bleeding was found after the incision, the gland was auto-transplanted into the sternocleidomastoid muscle.

Patients underwent subtotal parathyroidectomy according to a standard procedure that includes an anterior cervicotomy, bilateral neck exploration with identification of the 4 parathyroid glands and neuromonitoring of the recurrent laryngeal nerve by nerve integrity monitor (NIM) and occasionally by automatic periodic stimulation (APS, continuous). The search for ectopic glands was performed
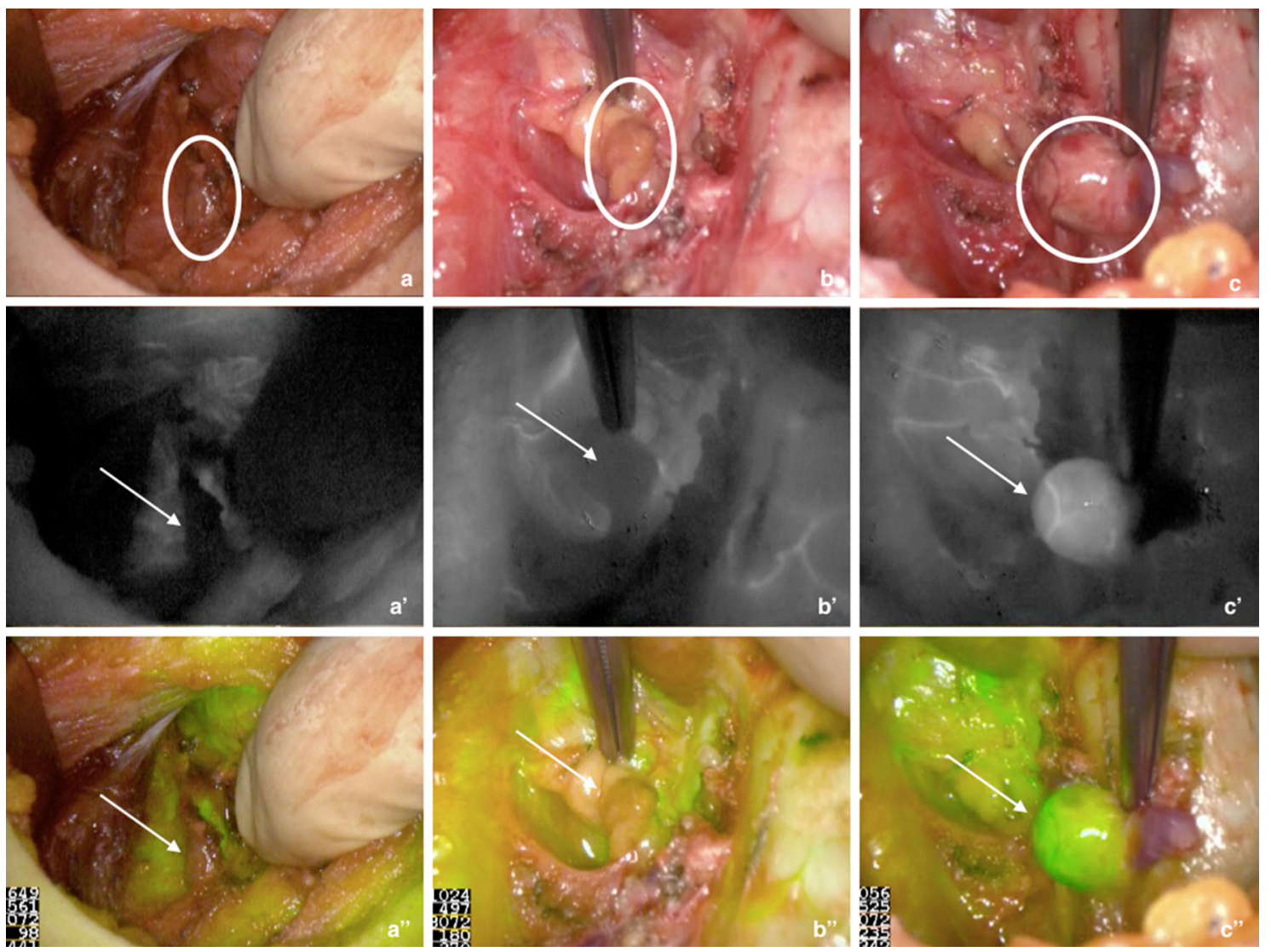

Fig. 1 Representative images of a devascularized (ICG score $=0$ ), b moderately well vascularized $(\mathrm{ICG}=1)$ and $\mathbf{c}$ well vascularized parathyroid gland (ICG $=2$ ) using indocyanine green angiography. $\mathbf{a}, \mathbf{b}, \mathbf{c}$ Normal view; $\mathbf{a}^{\prime}, \mathbf{b}^{\prime}, \mathbf{c}^{\prime}$ black and white near-infrared view; $\mathbf{a}^{\prime \prime}$, $\mathbf{b}^{\prime \prime}, \mathbf{c}^{\prime \prime}$ combined normal and near-infrared view. Circles and arrows indicate the parathyroid gland. $I C G$ indocyanine green 
according to known principles of parathyroid exploration (thymic or para-esophageal exploration depending on the gland missing).

Criteria such as anatomic localization, vascularization and morphological aspect of the glands were used to select which gland will be subtotally resected (ideally inferior, small, or the gland with no macroscopic nodule). Subtotal parathyroidectomy was performed by placing clips anterior to the resection site of the glands. Special attention was given to avoid placing clips on the parathyroid hilum. Prior to resection of the parathyroid glands, perfusion of the remaining remnant was controlled by injection of $3.5 \mathrm{~mL}$ of the ICG solution (Fig. 2).

These images show the level of perfusion of each remnant in between the clips and enable the choice of the best functional one. After final confirmation of a well-perfused
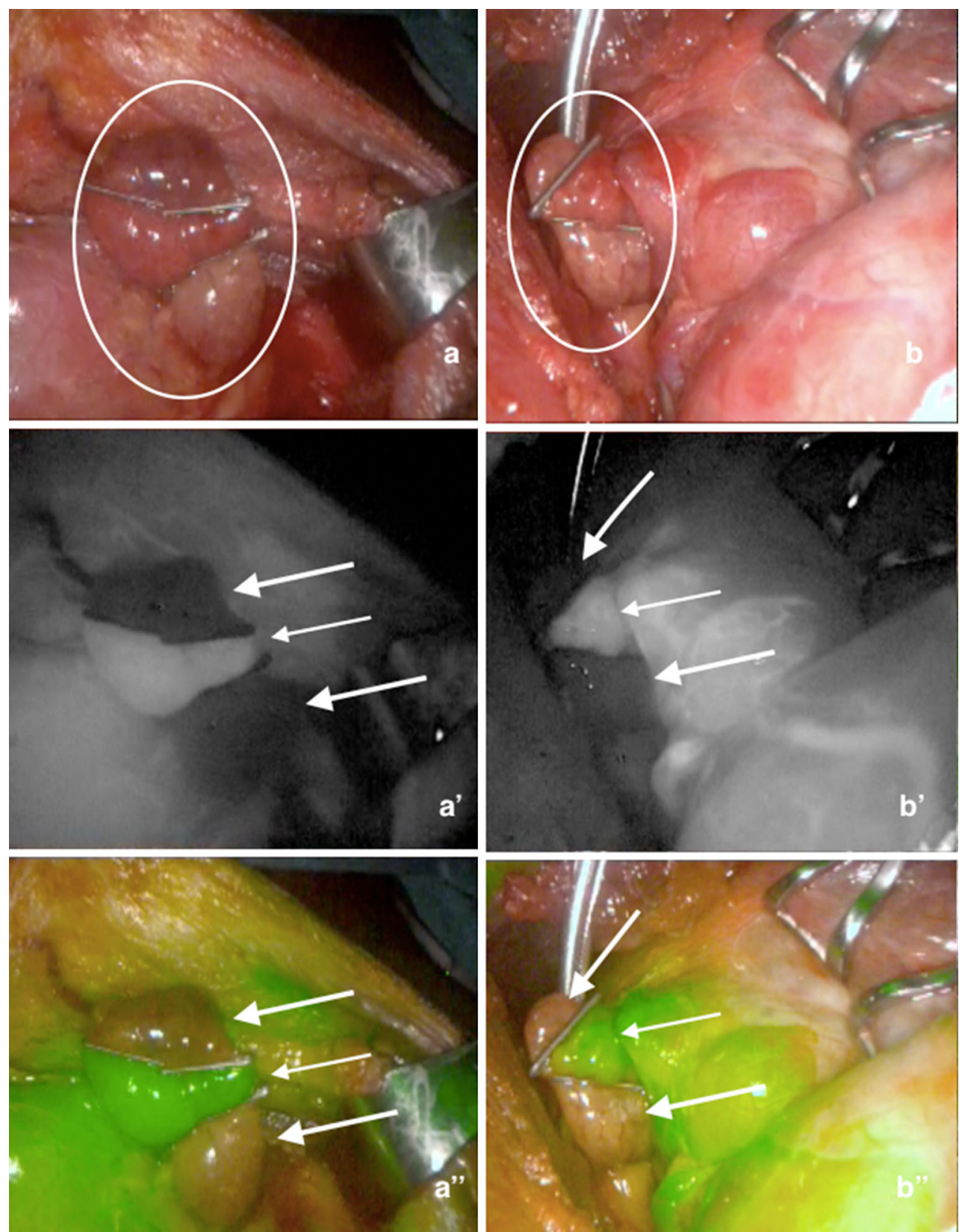

Fig. 2 Representative images of clipped parathyroid glands using indocyanine green angiography to evaluate remnants during subtotal parathyroidectomy in two separate cases. a, b Normal views; $\mathbf{a}^{\prime}, \mathbf{b}^{\prime}$ black and white near-infrared views; $\mathbf{a}^{\prime \prime}, \mathbf{b}^{\prime \prime}$ combined normal and

near-infrared views. Circles indicate parathyroid gland. Thin arrows indicate well-perfused remnant, thick arrows indicate devascularized parathyroid tissue 
remnant, the non-perfused parathyroid tissue can then be safely resected with scissors, and the other glands removed. Depending on the findings of surgery, a thymectomy was performed. The entire technique is shown in Video 4 and another example was published by our group [5].

Another use of ICG angiography is during thyroid surgery, to evaluate parathyroid gland function. Transient hypocalcaemia is frequent and has been described in $15-30 \%$ of patients; however permanent hypocalcaemia after thyroidectomy has been reported in 1-3\% of patients. Our group has performed a study to evaluate parathyroid gland function and to ascertain the absence of post-operative hypoparathyroidism in patients who underwent total thyroidectomy and in whom at least one well-perfused gland was found on ICG angiography [6]. These preliminary results showed that all 30 patients had PTH levels in the normal range on post-operative days 1 and 10. Furthermore, preliminary studies performed in nine patients who underwent subtotal parathyroidectomy and had confirmed well-perfused remnant by ICG angiography showed that their PTH levels dropped to $76 \pm 6.5 \%$ from the preoperative levels and that at follow-up, all of them had calcium and PTH levels in the normal range.

In conclusion, these findings suggest a correlation between parathyroid perfusion and post-operative parathyroid function. Further studies are needed to validate ICG angiography as a tool to assess the function of the parathyroid glands in real time during parathyroid and thyroid surgery. In the future, new applications with this technique will be widely used and performed in endocrine neck surgery to help surgeons with intra-operative decision-making.

\section{Compliance with ethical standards}

Conflict of interest The authors have no conflict of interests to declare.

\section{References}

1. Julian MT, Balibrea JM, Granada ML et al (2013) Intact parathyroid hormone measurement at 24 hours after thyroid surgery as predictor of parathyroid function at long term. Am J Surg 206:783-789

2. Kaczirek K, Riss P, Wunderer G et al (2005) Quick PTH assay cannot predict incomplete parathyroidectomy in patients with renal hyperparathyroidism. Surgery 137:431-435

3. de Boer E, Harlaar NJ, Taruttis A et al (2015) Optical innovations in surgery. Br J Surg 102:e56-e72

4. Schols RM, Connell NJ, Stassen LP (2015) Near-infrared fluorescence imaging for real-time intraoperative anatomical guidance in minimally invasive surgery: a systematic review of the literature. World J Surg 39:1069-1079. doi:10.1007/s00268-014-2911-6

5. Vidal Fortuny J, Guigard S, Diaper J et al (2015) Subtotal parathyroidectomy under indocyanine green angiography. J Video Endocrinol. doi:10.1089/ve.2015.0056

6. Vidal Fortuny J, Belfontali V, Sadowski S et al (2015) Parathyroid gland angiography with indocyanine green fluorescence to predict parathyroid function after thyroid surgery. Br J Surg. doi:10.1002/ bjs.10135 